University of Nebraska - Lincoln

DigitalCommons@University of Nebraska - Lincoln

Papers in the Earth and Atmospheric Sciences

Earth and Atmospheric Sciences, Department

2015

Polarimetric Tornadic Debris Signature Variability and Debris

Fallout Signatures

Matthew S. Van Den Broeke

Follow this and additional works at: https://digitalcommons.unl.edu/geosciencefacpub

Part of the Earth Sciences Commons

This Article is brought to you for free and open access by the Earth and Atmospheric Sciences, Department of at DigitalCommons@University of Nebraska - Lincoln. It has been accepted for inclusion in Papers in the Earth and Atmospheric Sciences by an authorized administrator of DigitalCommons@University of Nebraska - Lincoln. 


\title{
Polarimetric Tornadic Debris Signature Variability and Debris Fallout Signatures
}

\author{
MatThew S. VAN Den BRoeke \\ Department of Earth and Atmospheric Sciences, University of Nebraska-Lincoln, Lincoln, Nebraska
}

(Manuscript received 11 March 2015, in final form 9 October 2015)

\begin{abstract}
Values of polarimetric radar variables may vary substantially between and through tornadic debris signature (TDS) events. Tornadoes with higher intensity ratings are associated with higher average and extreme values of reflectivity factor at horizontal polarization $Z_{\mathrm{HH}}$ and lower values of copolar cross-correlation coefficient $\rho_{\mathrm{hv}}$. Although values of these variables often fluctuate through reported tornado life cycles, $Z_{\mathrm{HH}}$ repeatably decreases and $\rho_{\mathrm{hv}}$ repeatably increases across the volume scan immediately following reported tornado demise. Land cover has a relatively small effect on values of the polarimetric variables within TDSs, although near-radar urban TDSs may exhibit relatively high $Z_{\mathrm{HH}}$ values. TDS areal extent is typically larger aloft than near the surface, although this trend may reverse in the most intense tornadoes. Maximum altitude to which a TDS is visible is more strongly a function of tornado intensity than of land cover or ambient shear and instability. Debris often disappears once lofted but may also be observed to spread out downstream with the storm-relative flow or to fall out along the parent storm's northwest flank in a debris fallout signature (DFS). DFS characteristics, although variable, most commonly include $Z_{\mathrm{HH}}$ values of $30-35 \mathrm{~dB} Z, \rho_{\mathrm{hv}}$ values of $0.60-0.80$, and values of differential reflectivity $Z_{\mathrm{DR}}$ that are repeatably near $0 \mathrm{~dB}$.
\end{abstract}

\section{Introduction}

Tornadoes may loft substantial quantities of debris, and associated polarimetric tornadic debris signatures (TDSs) are well known and often observed. They can be identified by collocated low values of copolar crosscorrelation coefficient $\rho_{\mathrm{hv}}$, a pronounced radial velocity $V_{r}$ vortex, and often a local maximum in reflectivity factor at horizontal polarization $Z_{\mathrm{HH}}$ and low values of differential reflectivity $Z_{\mathrm{DR}}$ (e.g., Ryzhkov et al. 2005; WDTD 2011; Schultz et al. 2012; Bodine et al. 2013; Van Den Broeke and Jauernic 2014). Specific thresholds of the polarimetric variables have proven challenging to define, given, for example, precipitation entrainment (e.g., Bodine et al. 2011), a strong vortex within a weakreflectivity region (e.g., WDTD 2011), and land-cover variability (e.g., Van Den Broeke and Jauernic 2014).

Relatively high $Z_{\mathrm{HH}}$ values to $72 \mathrm{dBZ}$ have been reported in TDSs (Bunkers and Baxter 2011). The $Z_{\mathrm{HH}}$ in the TDS was originally required to be greater than $45 \mathrm{~dB} Z$ (Ryzhkov et al. 2005), using a sample of

Corresponding author address: Matthew S. Van Den Broeke, University of Nebraska-Lincoln, Earth and Atmospheric Sciences, 306 Bessey Hall, Lincoln, NE 68588-0340.

E-mail: mvandenbroeke2@unl.edu. supercell storms. Since then, the signature has been discovered in additional storm modes (e.g., Kumjian and Ryzhkov 2008; Schultz et al. 2012) and on occasion may also be associated with low reflectivity in the supercell echo appendage. Thus, guidelines given to National Weather Service (NWS) forecasters indicate a minimum $Z_{\mathrm{HH}}$ threshold of $20 \mathrm{dBZ}$, ensuring a signal-to-noise ratio that is high enough for good quality of the polarimetric variables (WDTD 2011).

The $\rho_{\mathrm{hv}}$ is the variable that is most helpful for debris detection when collocated with a vortex. Initially, $\rho_{\mathrm{hv}}$ values of less than 0.8 were required for a TDS (Ryzhkov et al. 2005). Entrainment of meteorological scatterers may, however, increase $\rho_{\mathrm{hv}}$ values (e.g., Schwarz and Burgess 2011; Bodine et al. 2011). Consequently, NWS training indicates $\rho_{\mathrm{hv}}$ values up to 0.95 associated with tornadic debris, provided that it represents a local $\rho_{\mathrm{hv}}$ minimum and is collocated with a strong vortex (WDTD 2011). Values of $Z_{\mathrm{DR}}$ are often near $0 \mathrm{~dB}$ in tornadic debris, but hydrometeor entrainment may raise values (e.g., Kumjian and Ryzhkov 2008) or scattering by particles that are large relative to the radar wavelength may lower values (Bodine et al. 2014), and therefore this variable is less helpful in distinguishing debris. Therefore, specific $Z_{\mathrm{DR}}$ values are no longer considered to be appropriate for identifying a 
TDS, but, when collocated with a strong vortex, sufficiently high $Z_{\mathrm{HH}}$, and sufficiently low $\rho_{\mathrm{hv}}$, low $Z_{\mathrm{DR}}$ values serve to increase confidence in the presence of debris (WDTD 2011). Promising approaches to eliminate the need for specific thresholds involve percentile values of the polarimetric variables (Bodine et al. 2013) and fuzzy-logic methods (Wang and Yu 2015).

In a climatological study of TDS characteristics, Van Den Broeke and Jauernic (2014) found several factors that influence observed TDS characteristics. TDSs were larger and present to higher altitude as tornado intensity and pathlength increased, and they were more common with tornadoes that were responsible for at least $\$ 1$ million in reported damage. Distance to the nearest radar was an important consideration, as were the primary land-cover classification that was affected and the time of year. For all tornadoes in the domain of a polarimetric radar from January 2012 to May 2013, 16\% were associated with a TDS (Van Den Broeke and Jauernic 2014).

The polarimetric TDS may also provide a way to learn about how debris behaves once it is ingested by a tornado. Debris ingestion may be important to tornado dynamics, reducing the associated pressure drop and slowing the near-surface wind (e.g., Lewellen et al. 2008). For stronger tornadoes, the tornado vortex may be observed to extend upward as a weak-echo column, which is attributed to hydrometeor centrifuging at low levels and strong vertical motion aloft (Tanamachi et al. 2012). Higher $Z_{\mathrm{HH}}$ values are often observed aloft at a slightly larger radius than the tornado and may spread out with height (e.g., Dowell et al. 2005); debris may also be ejected from the low-level vortex (Kurdzo et al. 2015). Trajectories of small debris elements have been calculated using the Hybrid SingleParticle Lagrangian Integrated Trajectory (HYSPLIT) model for the 27 April 2011 tornado outbreak (Knox et al. 2013). Few debris elements were documented to be transported downstream by tornadoes that on the enhanced Fujita scale were rated as category 2 (EF2) or weaker, whereas EF4 and EF5 tornadoes were responsible for the transport of most debris elements, including paper. Debris was seen to be transported as far as $353 \mathrm{~km}$, and, on average, debris tended to fall out $10^{\circ}$ to the left of the tornado motion vector, with larger debris elements falling out just to the right of the direction of tornado motion (Knox et al. 2013). Prior work has found that large debris elements fall out to the right of tornado motion while smaller debris elements are scattered in the direction of tornado motion (Davies-Jones et al. 1978). Debris may be deposited at the ground in a series of clusters (Magsig and Snow 1998), falling out with precipitation or outside precipitation regions. Debris has been documented to remain at lower elevation over urban land cover (Stelten and Wolf 2014; Van Den Broeke and Jauernic 2014), possibly because urban debris elements are larger and/or heavier. Such heavy debris elements are typically observed to fall out to the right of the tornado path, although most debris elements typically fall out along or to the left of the storm motion vector (Snow et al. 1995).

Prior studies have not, using a large sample of cases from an operational dataset, examined how the polarimetric radar variables change through the life cycle of the TDS and associated tornado. The effects of land cover and other pertinent factors have also not been broadly investigated. Polarimetric radar characteristics of vertical debris transport and debris fallout have not been commonly presented. Using a large sample of TDSs from 2012 and early 2013, this study addresses the following questions:

1) What are typical values of $Z_{\mathrm{HH}}$ and $\rho_{\mathrm{hv}}$ in TDSs, and how do they vary with tornado characteristics?

2) How do values of $Z_{\mathrm{HH}}$ and $\rho_{\mathrm{hv}}$ typically change through tornado and TDS life cycles?

3) What influence does land cover have on TDS characteristics?

4) How does fallout of suspended debris manifest, and where does it typically occur?

Quantification of values of polarimetric radar variables in TDSs under varying circumstances may be helpful to those who use TDSs operationally and will provide results that are useful to those who model TDS occurrence and characteristics. In addition, characteristics of debris fallout and vertical debris columns may be useful to the operational and research communities.

\section{Data and methods}

TDSs utilized for this study were the same as those identified by Van Den Broeke and Jauernic (2014). In summary, all reported tornadoes from 1 January 2012 to 0400 UTC 1 June 2013 were identified using the National Climatic Data Center's Storm Events Database. For each of the 1284 tornado events in the domain of a polarimetric Weather Surveillance Radar-1988 Doppler (WSR-88D), the radar data were examined for evidence of a TDS. TDSs were identified from 46 WSR-88D sites. Criteria for identifying TDSs included collocation of a rotational signature in $V_{r}, Z_{\mathrm{HH}} \geq 20 \mathrm{~dB} Z$, and $\rho_{\mathrm{hv}} \leq 0.8$. To account for precipitation ingestion, higher $\rho_{\mathrm{hv}}$ values were allowed as long as they were collocated with $Z_{\mathrm{HH}} \geq 30 \mathrm{~dB} Z$ and $Z_{\mathrm{DR}}$ near $0 \mathrm{~dB}$ and represented a local minimum within the TDS. In the initial study, 119 
TDSs were identified from a total of 744 individual tornado events (Van Den Broeke and Jauernic 2014). Here, events were eliminated if two reported tornadoes overlapped the same TDS, yielding 113 TDS events $(\mathrm{EF} 0=25, \mathrm{EF} 1=40, \mathrm{EF} 2=26, \mathrm{EF} 3=15$, and EF4 $5=7)$.

For each TDS, detailed information was recorded, including appearance and disappearance time, values of the polarimetric variables throughout the TDS life cycle, and TDS vertical and areal extent. Distance to the nearest radar was estimated as the distance to the reported track midpoint. These data allowed comparison of values of the polarimetric variables with reported tornado and TDS life cycles. To the degree possible, debris was tracked from ingestion to surface deposition, allowing an assessment of the signature associated with base-scan debris fallout.

Particularly pertinent to TDS characteristics may be the land cover over which a tornado travels. Land-cover information utilized in this study was identical to that reported in Van Den Broeke and Jauernic (2014). To summarize here, the U.S. Geological Survey (USGS) National Atlas of the United States (since retired: see http:// nationalmap.gov/small_scale/ and http://nationalmap.gov/ small_scale/mld/lancovi.html) was used to assign one or two dominant land-cover classifications for each TDS event. Land-cover data in the National Atlas of the United States have 200-m resolution and 21 land-cover classifications, which were remapped to seven land-cover categories on the basis of the dominant material being lofted (presented in Table 1). USGS land-cover classifications were compared with satellite imagery to ensure a minimal effect of land-cover change through time (Van Den Broeke and Jauernic 2014).

\section{Observations of polarimetric-variable values in TDSs}

\section{a. Typical values of the polarimetric variables in TDSs}

For all volume scans with a TDS, $Z_{\mathrm{HH}}$ and $\rho_{\mathrm{hv}}$ values were listed in association with all TDS pixels. This allowed the computation of quartiles of the $Z_{\mathrm{HH}}$ and $\rho_{\mathrm{hv}}$ values for each base scan. Quartiles were used as an outlier-resistant measure, given the statistical variability of values of the polarimetric variables and the varying number of pixels composing each TDS. In the results presented below, quartiles values discussed are the average of all quartile values for a given category (e.g., EF-scale rating).

The distribution of $Z_{\mathrm{HH}}$ values within TDSs was influenced by the tornado intensity rating (Fig. 1a). Median $Z_{\mathrm{HH}}$ increased steadily from $41.2 \mathrm{dBZ}$ for EF0
TABLE 1. The seven land-cover classifications used in this study, and the number of TDSs detected over each. Note that the sum of $n$ is greater than 113 because tornadoes could be assigned two landcover classifications.

\begin{tabular}{llr}
\hline \hline Land-cover classification & Predominant material lofted & $n$ \\
\hline Water & Water & 4 \\
Urban & Anthropogenic materials & 18 \\
Deciduous vegetation & Broad vegetative elements & 41 \\
Coniferous vegetation & Coniferous vegetative elements & 7 \\
Grassland & Grasses & 44 \\
Cropland & Crops and soil & 33 \\
Bare ground & Soil or rock & 0 \\
\hline
\end{tabular}

tornadoes to $46.3 \mathrm{~dB} Z$ for tornadoes rated $\mathrm{EF} 3$ and then decreased to $40.5 \mathrm{~dB} Z$ for tornadoes rated EF4/5. The relatively low $Z_{\mathrm{HH}}$ median value for EF4/5 tornadoes may be a result of the small sample size $(n=7)$ but may also be because these tornadoes tended to occur outside areas of precipitation. In general, for tornadoes that were not isolated from precipitation, higher $Z_{\mathrm{HH}}$ values indicated stronger tornadoes in this sample. The $\rho_{\mathrm{hv}}$ showed a similar pattern, with median values decreasing from $\sim 0.85$ for EF0-2 events to $\sim 0.76$ for EF4/5 events (Fig. 1b). Strong tornadoes accounted for most TDSs with median $\rho_{\mathrm{hv}}$ values $<0.80$. These results are similar to those of Bodine et al. (2013), who suggest that polarimetric-variable values may have some utility for assessing tornado intensity and damage in real time.

Tornado pathlength and width were not closely related to $Z_{\mathrm{HH}}$ and $\rho_{\mathrm{hv}}$ median values (not shown). Reported damage, although a flawed metric given the accuracy of reports, was weakly associated with expected $Z_{\mathrm{HH}}$ and $\rho_{\mathrm{hv}}$ trends. In general, as reported damage increased, median $Z_{\mathrm{HH}}$ also increased (Fig. 1c) and median $\rho_{\mathrm{hv}}$ decreased (Fig. 1d). The $Z_{\mathrm{HH}}$ values were notably higher for events producing $>\$ 100,000$ in reported damage, and $\rho_{\text {hv }}$ values did not markedly decline until tornadoes produced $>\$ 10$ million in reported damage. This result is consistent with greater debris availability for tornadoes associated with substantial damage. A higher proportion of the $>\$ 10$-million tornadoes also affected urban areas $(33 \%$ for $>\$ 10$-million tornadoes; $12 \%-18 \%$ for tornadoes in the other damage categories). Much overlap exists between damage categories, with numerous high-damage events producing relatively low $Z_{\mathrm{HH}}$ and high $\rho_{\mathrm{hv}}$ values.

Distance from the radar was investigated as a possible factor affecting $Z_{\mathrm{HH}}$ and $\rho_{\mathrm{hv}}$ values in TDSs. Median $Z_{\mathrm{HH}}$ generally did not appear to be very sensitive to the TDS-radar distance (Fig. 1e), although events close to and far away from the radar site tended to have slightly higher median $Z_{\mathrm{HH}}$. For tornadoes close to the radar, 

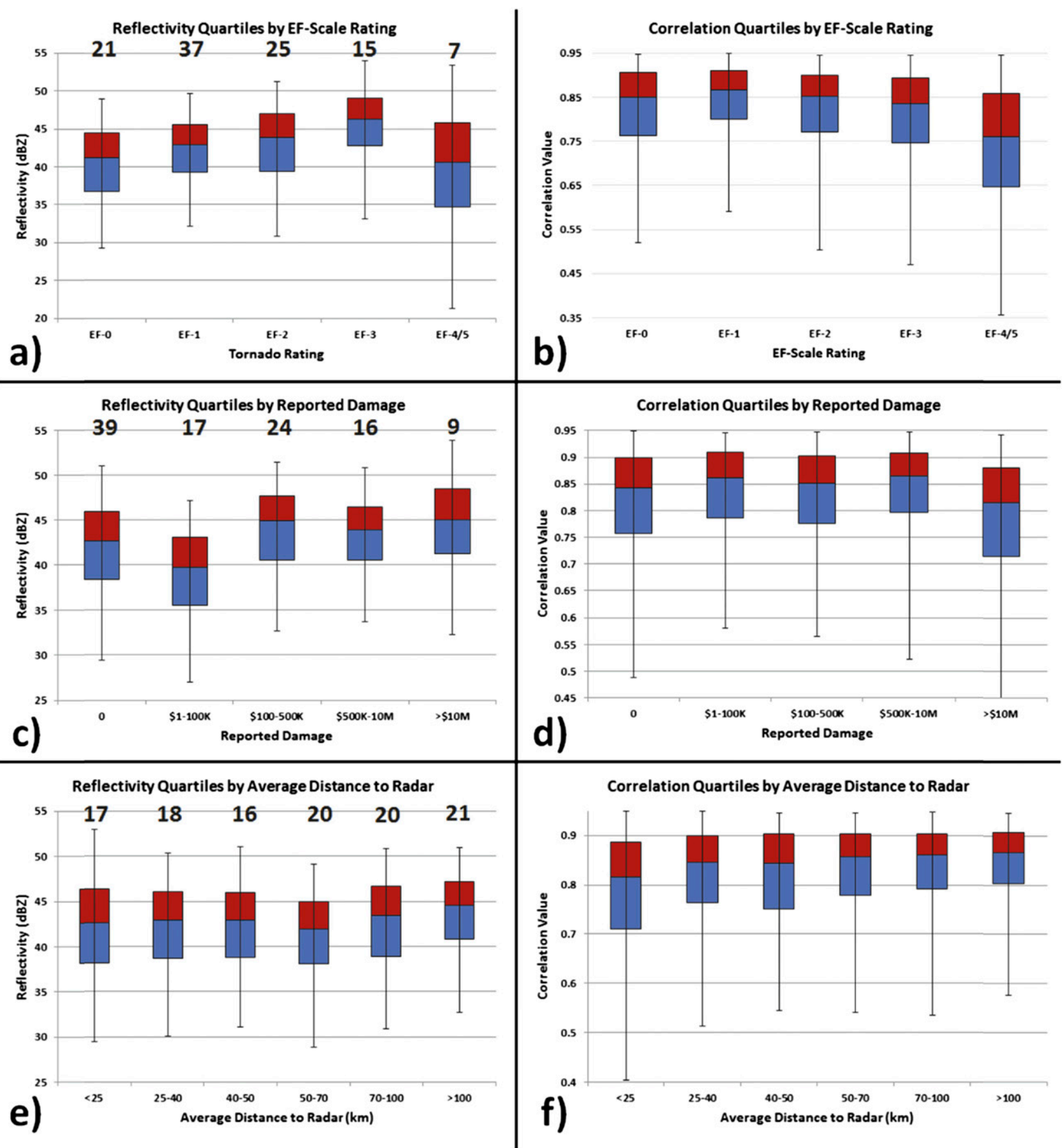

FIG. 1. Box-and-whisker plots of quartiles of $Z_{\mathrm{HH}}$ and $\rho_{\mathrm{hv}}$ values within TDSs vs tornado characteristics: the (a) $Z_{\mathrm{HH}}$ and (b) $\rho_{\mathrm{hv}}$ quartiles vs tornado intensity rating, the (c) $Z_{\mathrm{HH}}$ and (d) $\rho_{\mathrm{hv}}$ quartiles for reported damage categories, and the (e) $Z_{\mathrm{HH}}$ and (f) $\rho_{\mathrm{hv}}$ quartiles for categories of average distance from the radar. The red bar represents the third quartile, the blue bar represents the second quartile, and the red and blue bars are separated by the median value. The numbers at the top of (a),(c), and (e) indicate the number of cases contributing to each category.

this may be because resolution volumes are smaller there, allowing detection of small high- $Z_{\mathrm{HH}}$ areas. In addition, these volumes are at lower altitude and therefore are more likely to have a higher debris concentration and larger debris particles (e.g., Dowell et al. 2005). More-intense events tended to be observed farther from the radar, consistent with higher median $Z_{\mathrm{HH}}$ values at large range. The $\rho_{\mathrm{hv}}$ values did not appear to be sensitive to TDS-radar distance (Fig. 1f). 
TABLE 2. Median values of $Z_{\mathrm{HH}}$ and $\rho_{\mathrm{hv}}$ associated with all sample volumes during the ELC, MLC, and ND times of reported tornadoes, as defined in the text. Also shown are one-tailed WMW $p$ values. Comparisons that are significant at the $5 \%$ level are denoted with boldface font.

\begin{tabular}{lccc}
\hline \hline Stage & $n$ & $Z_{\mathrm{HH}}$ & $\rho_{\mathrm{hv}}$ \\
\hline ELC & 67 & 39.8 & 0.819 \\
MLC & 168 & 44.8 & 0.823 \\
ND & 140 & 42.0 & 0.859 \\
& Comparisons for $Z_{\mathrm{HH}}$ & & $p$ \\
\hline \multicolumn{3}{c}{ ELC vs MLC } & $\mathbf{0 . 0 0 4 5}$ \\
& ELC vs ND & & 0.1446 \\
MLC vs ND & & $\mathbf{0 . 0 4 9 5}$ \\
& Comparisons for $\rho_{\mathrm{hv}}$ & & $p$ \\
\hline & ELC vs MLC & & 0.2148 \\
& ELC vs ND & $\mathbf{4} \times \mathbf{1 0}$ \\
& MLC vs ND & $\mathbf{0 . 0 0 0 7}$ \\
\hline
\end{tabular}

\section{b. Polarimetric variations through tornado life cycles}

Temporal changes in $Z_{\mathrm{HH}}$ and $\rho_{\mathrm{hv}}$ values were investigated relative to reported tornado life cycles. First, for each event included in the database, the reported tornado lifespan was divided into early life cycle (ELC), mid-life cycle (MLC), and near-demise (ND) periods. ELC times were defined as minutes 1-6 after reported genesis, where minute 1 was the reported genesis time and all times prior to reported genesis. This period was chosen such that many ELC times may occur before substantial debris has been lofted to the altitude of the radar beam. It is unknown if the appearance of a TDS prior to reported genesis represents an increase in the low-level wind field that is lofting debris, the presence of an unreported tornado, incorrect reporting of tornado life cycle times, or other effects. MLC times were not assigned for events reported to last less than $8 \mathrm{~min}$ and extended until $1 \mathrm{~min}$ before reported demise for longer-duration events. The ND times were defined as all times from 1 min prior to reported demise onward, including all time periods postdemise. This period was chosen because of the operational value of knowing when a tornado has dissipated. Events with reported 2-min duration were removed from this analysis, unless a TDS lasted $\geq 3$ volume scans. In this case, reported genesis time and prior was defined as ELC and reported demise time onward was defined as ND. All radar scans within the defined ELC and MLC windows were examined for the presence of a TDS- $55.1 \%$ of possible ELC scans contained a TDS and $75.0 \%$ of possible MLC scans contained a TDS. Similar statistics were not meaningful for ND periods, since a TDS was often visible well after reported tornado demise.

The $Z_{\mathrm{HH}}$ and $\rho_{\mathrm{hv}}$ values varied through tornado life cycles. The median $Z_{\mathrm{HH}}$ within TDSs averaged $39.8 \mathrm{dBZ}$
TABLE 3. Median values of $Z_{\mathrm{HH}}$ and $\rho_{\mathrm{hv}}$ associated with all sample volumes during the first, middle, and last periods of a TDS, as defined in the text. Also shown are one-tailed WMW $p$ values. Comparisons that are significant at the $1 \%$ level are denoted with boldface font.

\begin{tabular}{|c|c|c|c|}
\hline Stage & $n$ & $Z_{\mathrm{HH}}$ & $\rho_{\mathrm{hv}}$ \\
\hline First & 59 & 41.7 & 0.832 \\
\hline Middle & 59 & 42.8 & 0.829 \\
\hline Last & 59 & 41.8 & 0.865 \\
\hline \multicolumn{2}{|c|}{ Comparisons for $Z_{\mathrm{HH}}$} & \multicolumn{2}{|c|}{$p$} \\
\hline \multicolumn{2}{|c|}{ First vs middle } & \multicolumn{2}{|c|}{0.3974} \\
\hline \multicolumn{2}{|c|}{ First vs last } & \multicolumn{2}{|c|}{0.3745} \\
\hline \multicolumn{2}{|c|}{ Middle vs last } & \multicolumn{2}{|c|}{0.4641} \\
\hline \multicolumn{2}{|c|}{ Comparisons for $\rho_{\mathrm{hv}}$} & \multicolumn{2}{|c|}{$p$} \\
\hline \multicolumn{2}{|c|}{ First vs middle } & \multicolumn{2}{|c|}{0.1335} \\
\hline \multicolumn{2}{|c|}{ First vs last } & \multicolumn{2}{|c|}{0.0026} \\
\hline \multicolumn{2}{|c|}{ Middle vs last } & \multicolumn{2}{|c|}{$\mathbf{0}$} \\
\hline
\end{tabular}

during ELC periods, increased to $44.8 \mathrm{~dB} Z$ during MLC periods, and decreased to $42.0 \mathrm{~dB} Z$ during ND periods (Table 2). The differences between MLC periods and ELC/ND periods were significant at level $p<0.05$, using a one-tailed Wilcoxon-Mann-Whitney (WMW) test of significance (Table 2). The increasing $Z_{\mathrm{HH}}$ values from the ELC to the MLC periods may indicate larger debris concentration during the MLC periods, whereas decreasing $Z_{\mathrm{HH}}$ in the ND periods likely indicates debris sedimentation. The median $\rho_{\mathrm{hv}}$ value remained nearly the same from the ELC period to the MLC period $(\sim 0.82)$, and these populations were not statistically different (Table 2). During ND periods, however, the median $\rho_{\mathrm{hv}}$ value increased to $\sim 0.86$, and this increase was statistically significant at $p<0.0001$ when compared with the ELC and MLC periods (Table 2). These observations are consistent with debris sedimentation through the ND period and are generally consistent with the findings of Ryzhkov et al. (2005) and Bodine et al. (2013) for smaller samples of TDSs.

Median $Z_{\mathrm{HH}}$ and $\rho_{\mathrm{hv}}$ values were also investigated relative to the first, middle, and last radar scans during which a TDS was visible. If a TDS was visible for an even number of radar scans, values of the polarimetric variables during the middle two time periods were averaged to obtain the "middle" value. The results are shown in Table 3. Median $Z_{\mathrm{HH}}$ did not change significantly across the reported tornado life cycle. This may be because some tornadoes exhibited increasing $Z_{\mathrm{HH}}$ toward demise as the tornado became embedded within precipitation whereas others exhibited decreasing $Z_{\mathrm{HH}}$ toward demise as a result of debris sedimentation. The $\rho_{\text {hv }}$ values were more variable, with a median near 0.83 during the first and middle scans in which a TDS was visible, increasing to $\sim 0.87$ in the last scan. Variability 
Reflectivity Quartiles near Tornado Demise

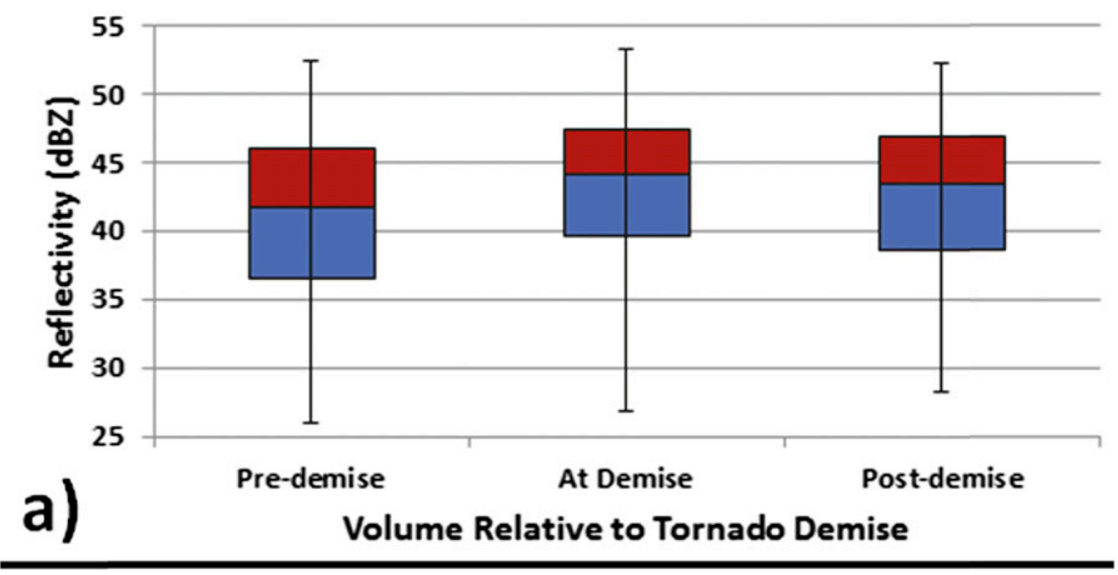

\section{Correlation Quartiles near Tornado Demise}

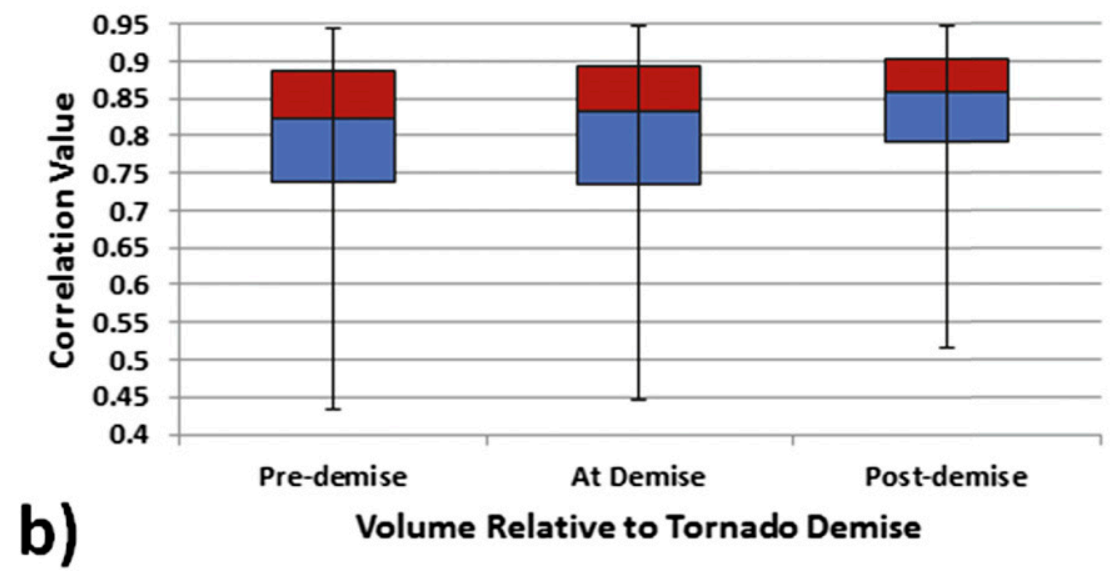

FIG. 2. As in Fig. 1, but for quartiles of (a) $Z_{\mathrm{HH}}$ and (b) $\rho_{\mathrm{hv}}$ near the reported tornado demise time. The "Pre-demise" label denotes the volume scan immediately before the reported tornado demise, the "At Demise" label denotes during or within 2 min of demise, and the "Postdemise" label denotes the volume scan immediately after demise.

between the last and first/middle periods was significant at $p<0.003$ using a WMW test (Table 3 ). These observations show the expected trends if debris is sedimenting after tornado demise. These results indicate that $\rho_{\mathrm{hv}}$ may be more useful in an operational setting for inferring the life cycle of strong tornadoes, although $Z_{\mathrm{HH}}$ trends may also be useful in some situations, as indicated by Bodine et al. (2013).

Of fundamental interest is whether polarimetric characteristics of the debris change noticeably once the volume of debris being added has decreased around the time of tornado demise. To investigate associated trends, volume scans were identified that were after but within 2 min of reported tornado demise. Then, 26 cases were identified that had such a near-demise scan, one scan before, and one scan after. Comparisons were made of these before-, during-, and after-demise times. The $Z_{\mathrm{HH}}$ values did not change notably after the demise time (Fig. 2a), although most $Z_{\mathrm{HH}}$ values of $>60 \mathrm{dBZ}$ were confined to the scans before and during demise. The $\rho_{\mathrm{hv}}$ values increased markedly (by $0.03-0.04$ ) in the postdemise volume scan $(p<0.02$ when compared with predemise and near-demise scans; Fig. 2b). Again, repeatable changes in $\rho_{\mathrm{hv}}$ values across tornado demise may have operational utility.

Also of interest is TDS occurrence relative to the reported tornado life cycle. Although volume scans average 4-5 min in length, across all cases on average a TDS appeared $4.4 \mathrm{~min}$ after reported tornadogenesis and disappeared $2.6 \mathrm{~min}$ after reported tornado demise. Large variations were present in this sample of cases (Fig. 3). For a subset of events ( $n=12$ of 117 total events 


\section{a) TDS Appearance Relative to Reported Genesis}

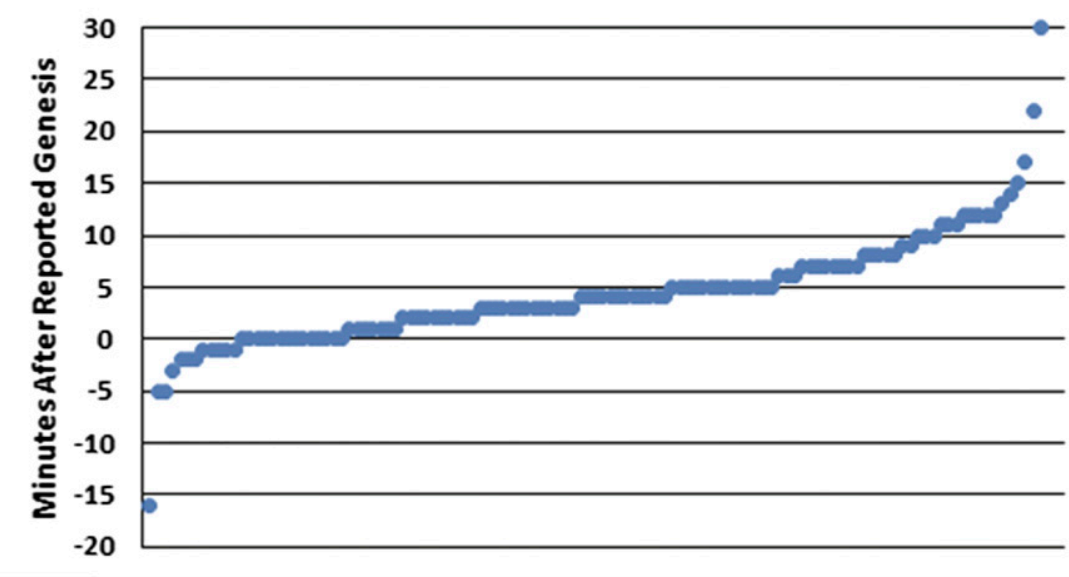

b)

TDS Disappearance Relative to Reported Demise

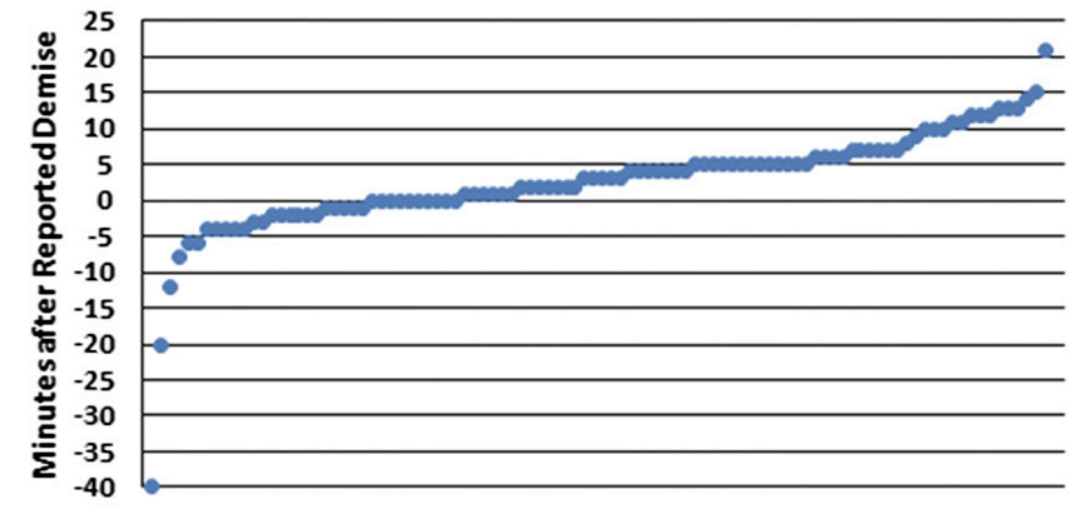

\section{TDS Events}

FIG. 3. TDS occurrence vs reported tornado life cycles: (a) the first appearance time of each TDS relative to reported tornadogenesis and. (b) the disappearance time of each TDS relative to reported tornado demise. Each blue dot represents one TDS event.

available for this analysis; $10.3 \%$ ), a TDS appeared prior to reported tornadogenesis (negative time values in Fig. 3a). These early-appearing events did not seem biased toward a particular time of day or convective mode. Intensity may have been a contributing factor-whereas 4 of $12(33 \%)$ of early-appearing cases were rated EF3+, only $17 \%$ of other events were as intense. Stronger tornadoes may be more likely on days with stronger lowlevel winds, in which more debris may become lofted as the wind field strengthens prior to tornadogenesis. Saari et al. (2014) provide other possible explanations for why a TDS may appear prior to reported tornadogenesis. In $24.5 \%$ of events, a TDS disappeared before tornado demise (negative time values in Fig. 3b). It was uncommon for a TDS to persist for more than $10 \mathrm{~min}$ after reported tornado demise, but in a few extreme events a TDS was present $15-20 \mathrm{~min}$ postdemise (Fig. 3b). The subset of events for which a TDS disappeared prior to tornado demise was slightly less likely to be rated $\mathrm{EF} 3+$, but this difference was not statistically significant. These results may be affected by, for example, errors in reported tornado times arising from poor reporting or tornado verification.

\section{c. Land-cover and polarimetric-variable values in $T D S s$}

Variability in $Z_{\mathrm{HH}}$ and $\rho_{\mathrm{hv}}$ values was investigated within TDSs as a function of land cover. TDS events were divided on the basis of the land-cover categories defined in Table 1. Variability between land-cover 
TABLE 4. Quartile values of $Z_{\mathrm{HH}}$ and $\rho_{\mathrm{hv}}$ in TDSs over various land-cover classifications. Here, $n$ denotes the number of individual tornado events contributing to the statistics for each land-cover classification, and Q1 and Q3 indicate the first and third quartiles, respectively.

\begin{tabular}{lrrrrrrrr}
\hline \hline & & \multicolumn{3}{c}{$Z_{\text {HH }}$ quartiles } & & \multicolumn{3}{c}{$\rho_{\text {hv }}$ quartiles } \\
\cline { 3 - 5 } \cline { 7 - 8 } Land cover & $n$ & Q1 & Median & Q3 & & Q1 & Median & Q3 \\
\hline Water & 4 & 41.2 & 45.4 & 47.4 & & 0.783 & 0.856 & 0.901 \\
Urban & 16 & 38.5 & 42.7 & 45.3 & & 0.778 & 0.858 & 0.905 \\
Deciduous & 38 & 39.1 & 43.5 & 46.9 & & 0.754 & 0.844 & 0.900 \\
Coniferous & 5 & 36.3 & 40.4 & 43.6 & & 0.799 & 0.865 & 0.911 \\
Grassland & 39 & 36.9 & 41.5 & 44.9 & & 0.761 & 0.843 & 0.898 \\
Cropland & 28 & 40.0 & 43.7 & 46.8 & & 0.762 & 0.843 & 0.897 \\
\hline
\end{tabular}

classes was investigated and controlled for storm mode and tornado intensity. Given the relatively small samples of events over some land-cover classifications (Table 1), results presented here should be considered to be preliminary.

Median $Z_{\mathrm{HH}}$ values were highest over deciduous forest, water, and cropland (Table 4), although events over water also spent considerable time over forest. These results were not statistically significant and warrant additional work. Median $Z_{\mathrm{HH}}$ was lowest over urban, grassland, and coniferous-forest land cover. A low median $Z_{\mathrm{HH}}$ over urban land cover is consistent with prior results (e.g., Stelten and Wolf 2014; Van Den Broeke and Jauernic 2014) and likely indicates large, heavy debris elements that sediment out more rapidly, but the difference between urban and other land-cover classes was not significant. Median $\rho_{\mathrm{hv}}$ values were lowest over grassland and cropland (Table 4), although these results were not significant at $p<0.05$. The highest median $\rho_{\mathrm{hv}}$ was seen over coniferous forest (Table 4). Additional cases should be examined to see if these preliminary patterns are repeatable.

Events were simplistically controlled for convective mode by assessing the percentage of TDS events over each land-cover classification associated with supercell thunderstorms. For our purposes, a supercell thunderstorm was defined as an organized convective cell with rotation evident in the radial velocity field within midlevels (2-6 km above radar level), as long as this rotation was coincident with the storm's updraft (e.g., with the area of relatively high values of differential reflectivity extending upward from low levels; Kumjian and Ryzhkov 2008). Across the entire dataset, $79.2 \%$ of TDSs were associated with supercell storms. This value was closely matched in the deciduous and cropland categories $(76.9 \%$ and $78.1 \%$ of events, respectively). Only $64.7 \%$ of urban TDSs were associated with the supercell mode, indicating that nonsupercell tornadoes are more likely to produce a TDS over urban areas. This seems to be because weaker tornadoes may produce a TDS in urban areas, particularly if close to the radar, and likely also reflects the bias of WSR-88Ds to be located near urban areas. Over grassland, a large proportion (90.2\%) of TDS events were produced by supercell storms; this result indicates the Great Plains bias of the grassland land-cover classification as well as the increased occurrence of the supercell mode in the Great Plains region (e.g., Smith et al. 2012). All four TDSs over primarily water and $66.6 \%$ of TDSs over coniferous forest were associated with supercells, although the sample sizes in these categories were too small to indicate definitive results.

A control was also applied for the intensity of tornadoes over each land-cover classification. Since damage indicators vary by land-cover classification, it may be more difficult to get strongly rated tornadoes over certain land-cover types; the results below should be interpreted in this context. For the large sample of reported tornadoes investigated by Van Den Broeke and Jauernic (2014), the percentage of tornadoes over each land-cover classification rated EF2 + was calculated and plotted against median values of $Z_{\mathrm{HH}}$ and $\rho_{\mathrm{hv}}$. For median $\rho_{\mathrm{hv}}$, little trend was noted-similar values occurred across a wide range of values for percentage of EF2+ tornadoes (Fig. 4a), regardless of land cover. Median $Z_{\mathrm{HH}}$ did vary by land cover, but these variations seemed to be related to the percentage of strong tornadoes over a given land-cover classification (Fig. 4b). Median $Z_{\mathrm{HH}}$ was largest in magnitude over water and lowest when a significant portion of the tornado track was over coniferous forest. Tornadoes with substantial track length over water appeared to be lofting debris while over land and keeping it suspended over water. It is also possible that reported beginning and ending latitude/ longitude and/or land-cover classifications may introduce slight error. Further evidence for or against the apparent association between $\rho_{\mathrm{hv}}$ values and percent of strong tornadoes may be obtained from a larger TDS dataset.

Polarimetric characteristics of a TDS may change with the underlying landcover. Two examples are shown here, one of a tornado affecting an urban area and the other of a tornado over primarily natural landcover. The Moore, Oklahoma, EF5 tornado of 20 May 2013 (e.g., Atkins et al. 2014) was chosen to reflect impacts of an urban area on TDS characteristics. This tornado produced a damage track that started over cropland and grassland, continued over heavily urbanized portions of Moore, Oklahoma, and ended east of Moore in an area of mixed grassland and deciduous forest (Fig. 5). A large portion of the damage track experienced consistent EF4 damage, with scattered EF5 damage over the city of 


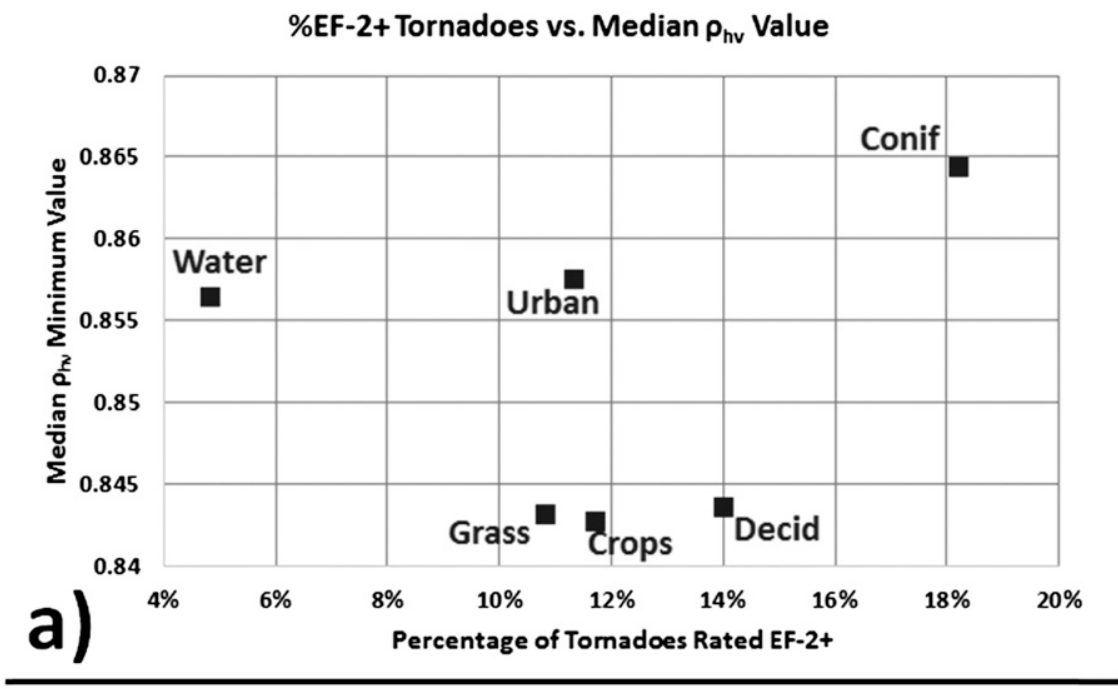

\%EF-2+ Tornadoes vs. Median $\mathrm{Z}_{\mathrm{HH}}$ Value

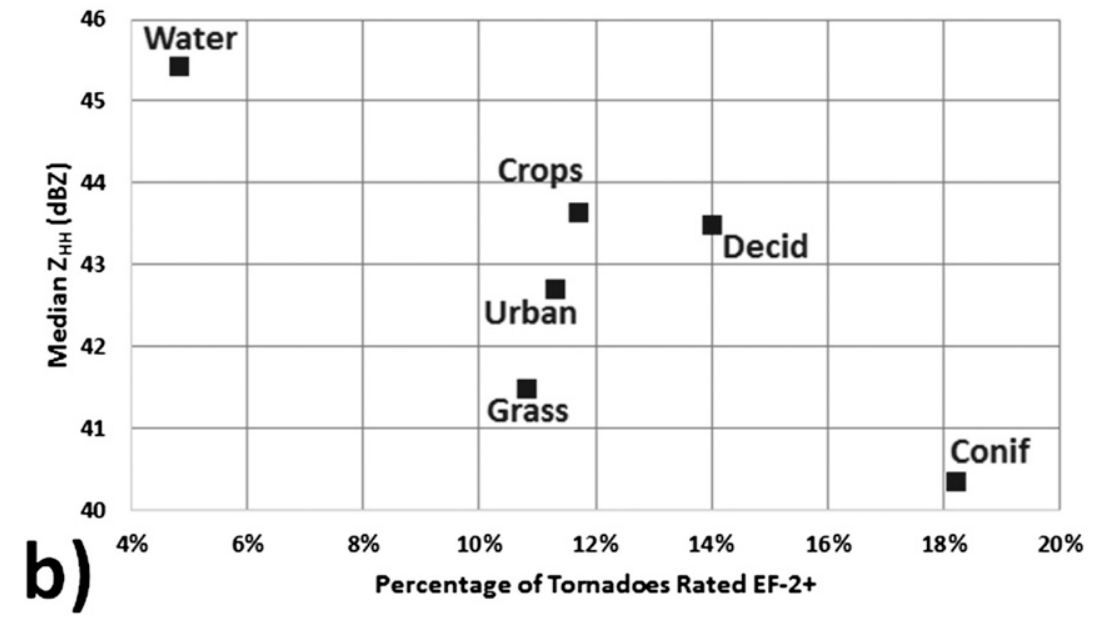

FIG. 4. Percentage of tornadoes rated EF2 + for each land-cover category vs the median (a) $\rho_{\mathrm{hv}}$ and (b) $Z_{\mathrm{HH}}$ value in TDSs.

Moore. For much of its track, the tornado was $\sim 20 \mathrm{~km}$ from the nearest WSR-88D, allowing excellent low-level sampling of associated debris. A well-defined TDS was first observed at the same time as reported tornadogenesis, although a $Z_{\mathrm{HH}}$ increase and a $\rho_{\mathrm{hv}}$ decrease were noted prior to reported tornadogenesis (Saari et al. 2014). Maximum $Z_{\mathrm{HH}}$ in the TDS rapidly increased to near $70 \mathrm{~dB} Z$, possibly indicating debris lofted from an area of EF4 damage early along the track (Fig. 5; Atkins et al. 2014). $Z_{\mathrm{HH}}$ dropped slightly but then increased to near $70 \mathrm{~dB} Z$ again while the tornado was producing EF4+ damage over Moore. The highest $Z_{\mathrm{HH}}$ for the event roughly corresponds to the time during which sporadic EF5 damage occurred. The $Z_{\mathrm{HH}}$ declined once the tornado was no longer over urban land cover, which is possibly partially related to the weakening observed at this point, and then declined markedly after reported tornado demise (Fig. 5). Minimum $\rho_{\text {hv }}$ within the TDS remained 0.208 (the minimum value recorded by WSR88D radars, although lower values are possible) throughout the time when the TDS was visible, perhaps because a large amount of anthropogenic debris was present from near the time of tornadogenesis (inferred from the presence of EF4 damage near the point of genesis; Fig. 5).

A case was also examined for which a tornado passed over primarily forest and pasture (a grassy land-cover type). This tornado, occurring in west-central Georgia, was rated EF2, although it produced EF1 damage over most of the track (Fig. 6). The $Z_{\mathrm{HH}}$ maxima within the TDS were relatively low over the first radar scans with a TDS, perhaps reflecting the weak intensity rating at this 


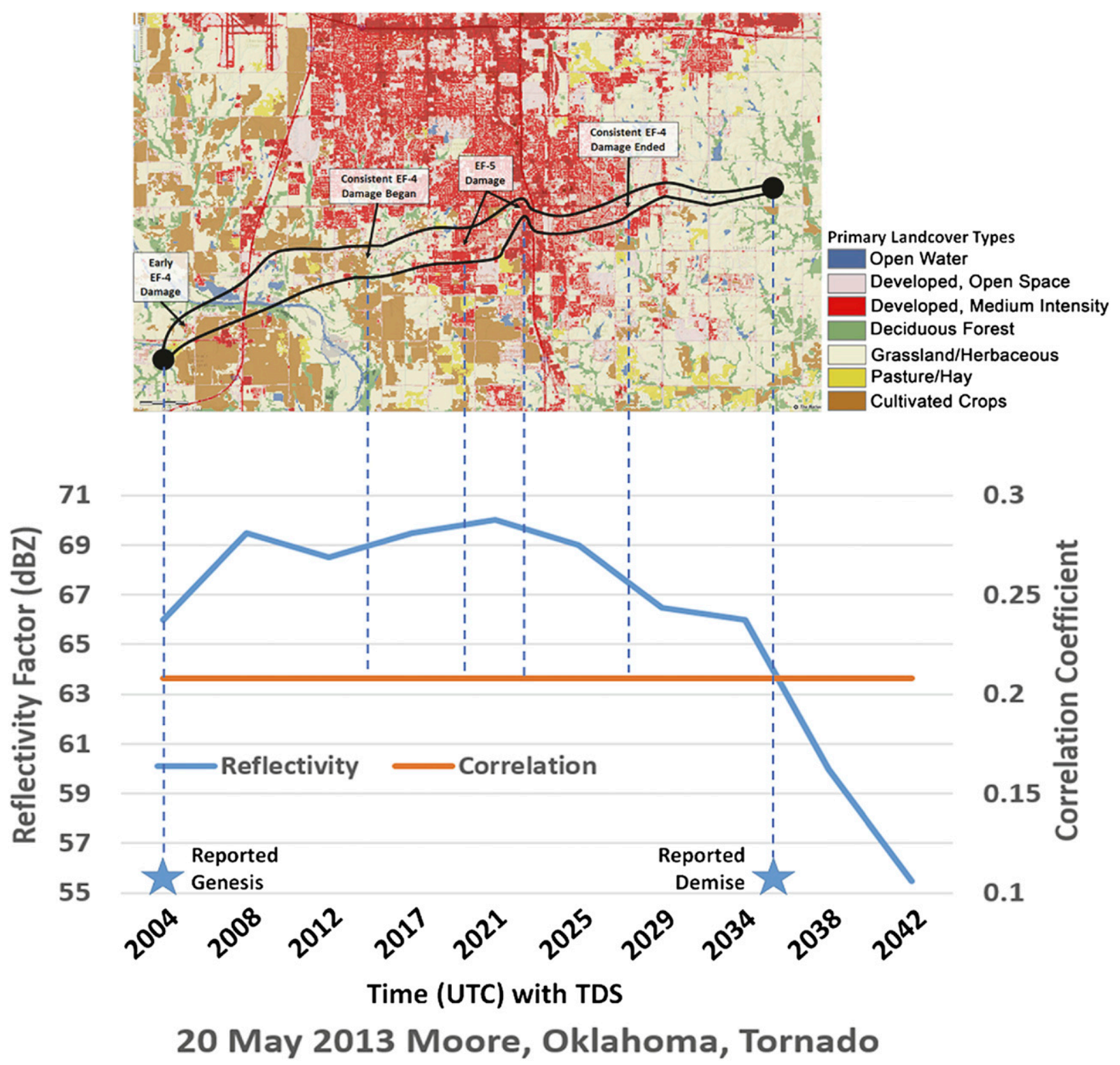

FIG. 5. (top) Track of the 20 May 2013 EF5 Moore tornado (black outline) overlaid on land cover. Brown colors represent cropland, pink (red) represent light (dense) urban, tan represents grassland, and green represents deciduous land cover. (bottom) Time series that indicates maximum $Z_{\mathrm{HH}}$ (blue line) and minimum $\rho_{\mathrm{hv}}$ (orange line) within the TDS at base-scan level for each sample volume. The time axis does not necessarily correspond directly to time/location along the tornado track, which would assume a constant tornado translation speed. The tornado path is adapted from the NWS Internet page "The Tornado Outbreak of May 20, 2013" (http://www.srh.noaa.gov/oun/ ?n=events-20130520).

time. The tornado was also passing over mostly coniferous forest through this time (dark green shading in Fig. 6), which has been associated with lower $Z_{\mathrm{HH}}$ maxima than other land-cover types. As the tornado became more intense, and after it had moved over an area of primarily pasture and deciduous forest, the $Z_{\mathrm{HH}}$ maxima increased markedly (Fig. 6). Minimum $\rho_{\text {hv }}$ within the TDS fluctuated from 0.208 to just over 0.80 . It is unknown why $\rho_{\mathrm{hv}}$ values started very low, but a minimum during the fourth and fifth samples may be related to the tornado's passage over some urbanized land cover along a roadway just prior (Fig. 6). Increasing $\rho_{\text {hv }}$ marked the postdemise period, as is typically observed.

\section{TDS spatial changes with height}

The vertical extent to which tornadic debris is observed may vary as a function of land cover. For 


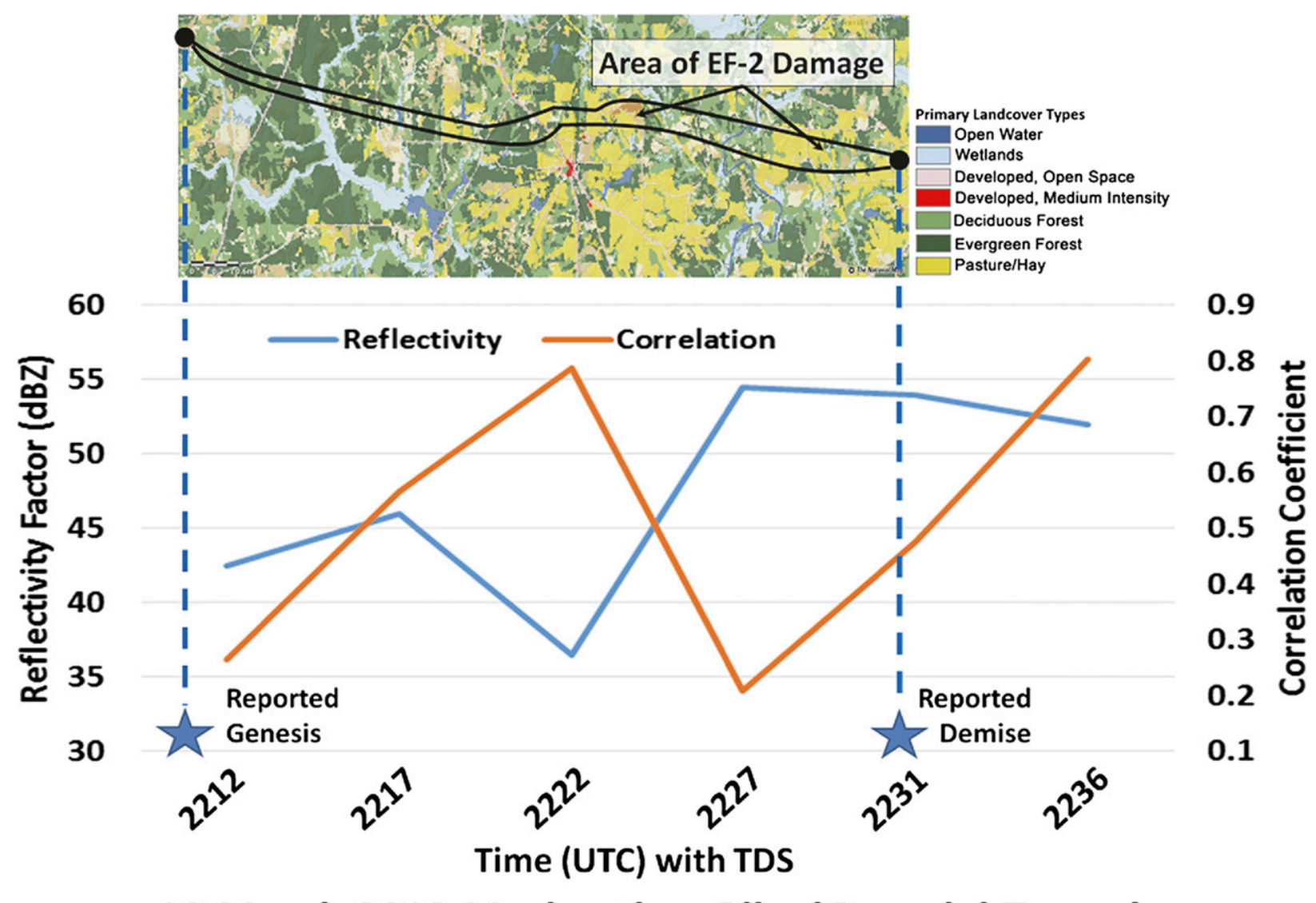

\section{March 2013 Meriwether-Pike (Georgia) Tornado}

FIG. 6. As in Fig. 5, but for the 18 Mar 2013 EF2 Meriwether-Pike Counties (Georgia) tornado. The tornado path is adapted from the NWS Internet page "Widespread Severe Weather Including Isolated Tornadoes Strike [sic] North and Central Georgia" (http://www.srh. noaa.gov/ffc/?n=severestorms_20130318).

example, a TDS over urban land cover is typically not observed to as high of an altitude (Stelten and Wolf 2014; Van Den Broeke and Jauernic 2014), possibly indicating the bias of WSR-88D locations near urban areas. A TDS may also remain at lower altitude if embedded in a downdraft (Bodine et al. 2013). Higheraltitude debris has been found in tornadoes with a higher intensity rating, with greater longevity, and with a larger radius of strong wind (Bodine et al. 2013; Van Den Broeke and Jauernic 2014).

The areal extent of the TDS was examined at various altitudes. The altitude at the center of the TDS was recorded for each scan at each elevation angle with a TDS, assuming standard beam propagation following the $4 / 3$ Earth radius model. A TDS areal extent was associated with each altitude value, and all data points were sorted by altitude into $0.5-\mathrm{km}$ bins (altitudes of $<0.5,0.5-1 \mathrm{~km}$, etc.). The TDS areal extent steadily increased with altitude (Fig. 7a), indicating a tendency toward spreading of debris aloft and the typically larger size of the vortex moving upward. The median value of TDS areal extent increased from $\sim 2 \mathrm{~km}^{2}$ for TDSs below $0.5 \mathrm{~km}$ in altitude to $\sim 7.5 \mathrm{~km}^{2}$ for TDSs at 4-4.5-km altitude (Fig. 7a). Above 4.5-km altitude, too few data points were present to calculate these statistics.

TDS areal extent was also a function of tornado intensity rating. Tornadoes that were rated higher on the EF scale tended to have larger TDS areal extent (Fig. 7b). Areal extent also increased with altitude for most EF-scale ratings, as seen for the entire dataset, but decreased with altitude for EF4/5 tornadoes (Fig. 7b). EF0 tornadoes typically had smaller areal extent, especially above 1-km altitude, although EF0, EF1, and EF2 tornadoes had relatively similar areal extent at most altitudes examined. TDSs associated with EF3 tornadoes were larger at all altitudes examined, and EF4/5 tornadoes typically had much larger TDSs (Fig. 7b). The sample size for EF4/5 tornadoes is small by the time the $2-2.5-\mathrm{km}$ altitude bin is reached $(n=7)$, possibly 
TDS Areal Extent by TDS Elevation

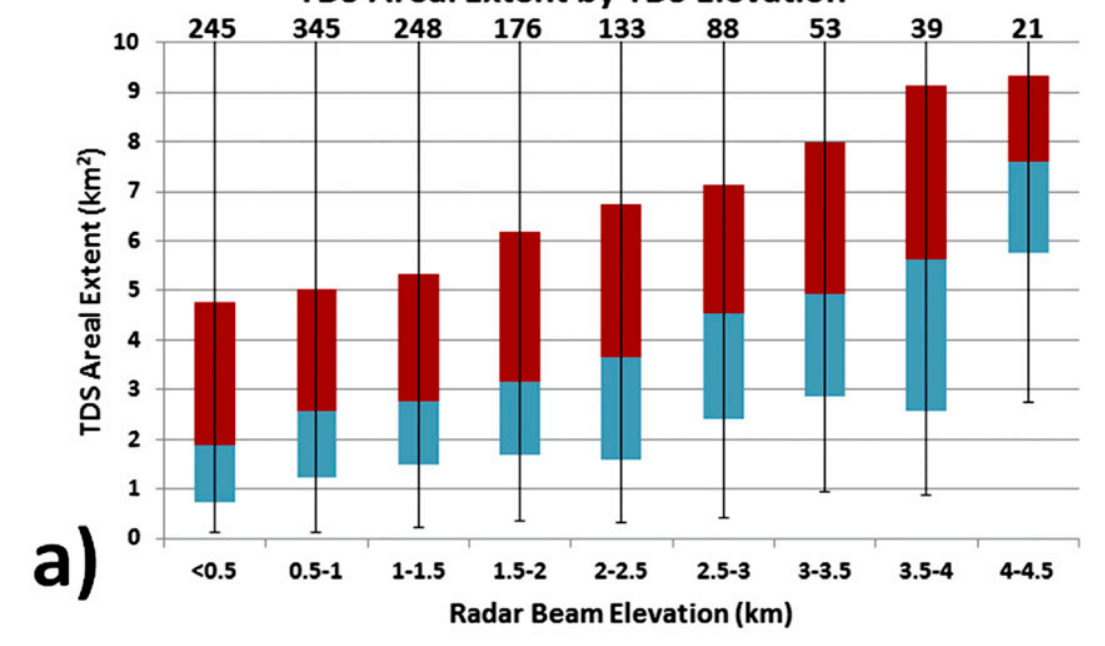

TDS Areal Extent vs. Elevation for Tornadoes of Varying EF-Scale Ratings

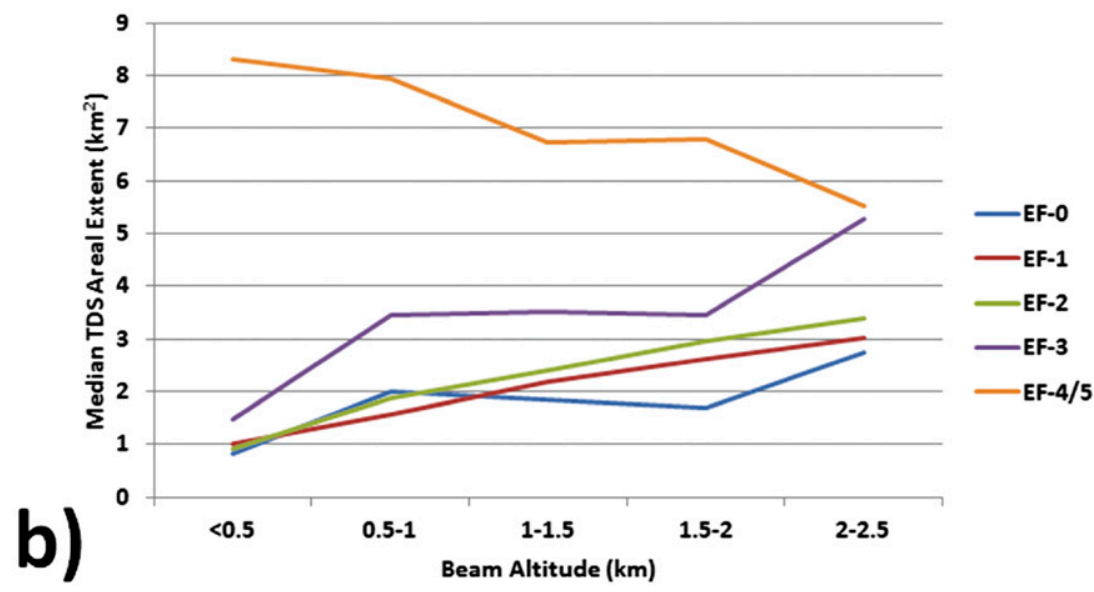

FIG. 7. (a) As in Fig. 1, but for the TDS areal extent as a function of radar-beam altitude, assuming a $4 / 3$ Earth radius model of beam propagation. The upper part of the whiskers is excluded because it would have distorted the vertical scale. The numbers at the top indicate the number of samples contributing to each set of statistics. (b) The median areal extent of TDSs as a function of radar-beam altitude for tornadoes of different intensity ratings.

accounting for some of the decrease in that bin, although $n \geq 30$ for all other points in Fig. $7 \mathrm{~b}$.

\section{The debris fallout signature}

Once debris is ingested and becomes visible in radar data, it is not always clear where the debris goes once the TDS disappears. Prior work has suggested that this debris often falls out just to the right or left of the tornado track depending on debris element size, but a large variety of fallout locations have been observed (e.g., Snow et al. 1995). This dataset of TDS events was examined to see whether repeatable manifestations of debris not associated with a TDS could be discovered.

In $\sim 10 \%$ of TDS events, debris was observed to spread out aloft and disperse downstream with time from the initial TDS position. An example of this behavior is provided in Fig. 8, showing a large debrisconsistent area (Figs. 8a,c) within and downstream of the associated vortex (Figs. 8b,d). In such cases, the debris is still obviously connected to the TDS and typically spreads out in approximately the direction of the ambient wind vector at the altitude of dispersion. When this occurs, values of the polarimetric variables are generally similar within the entire region of debris, 

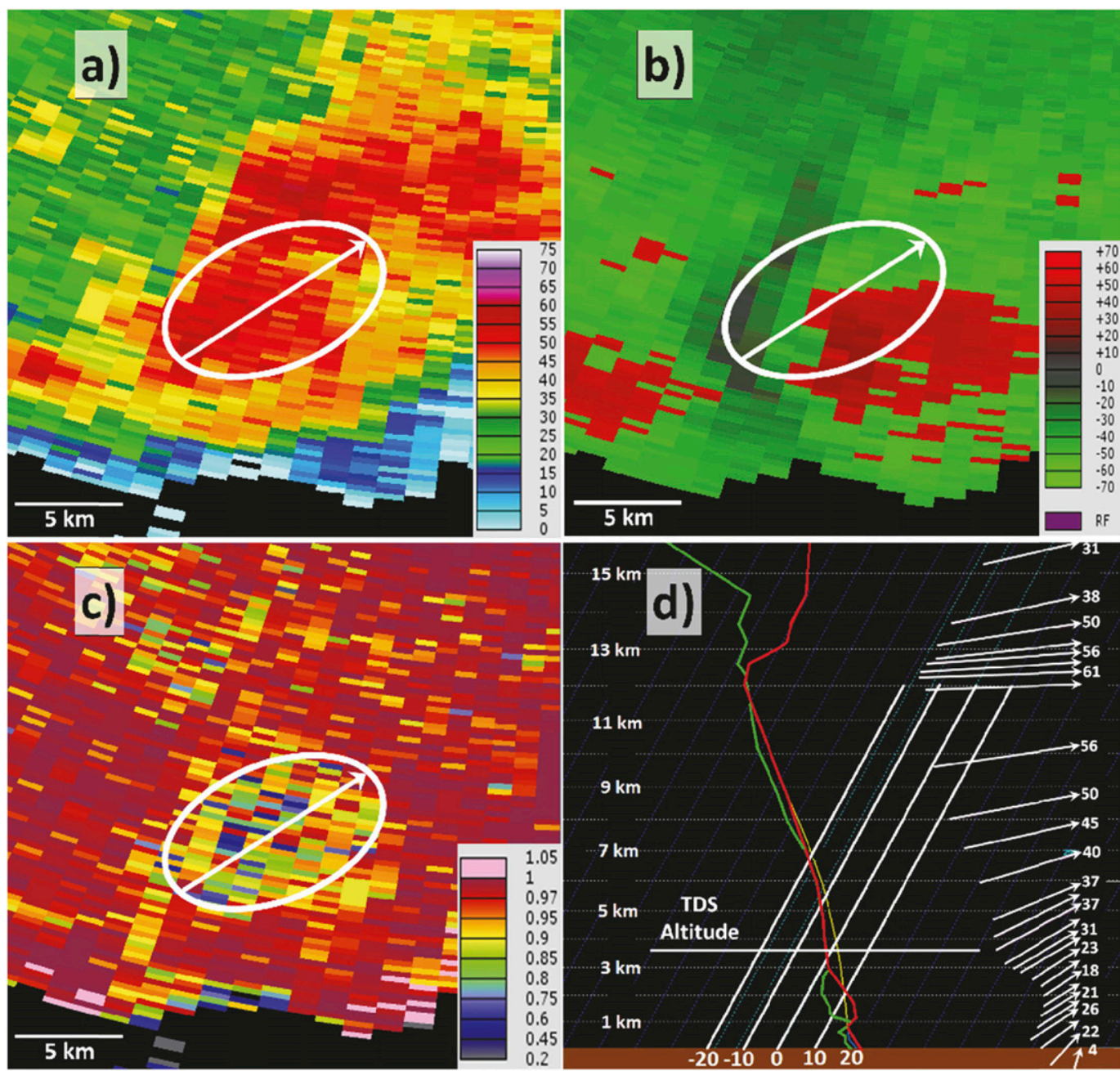

FIG. 8. Example of debris spreading downstream from the associated vortex, from the Birmingham, Alabama, WSR-88D (KBMX) at 0331 UTC 3 Mar 2012 (elevation angle 3.18 ): (a) $Z_{\mathrm{HH}}$ (dBZ), (b) radial velocity $(\mathrm{kt} ; 1 \mathrm{kt} \approx$ $0.51 \mathrm{~m} \mathrm{~s}^{-1}$ ), which is aliased (the red colors in the southeastern portion of the oval represent strong inbound velocities), (c) $\rho_{\mathrm{hv}}$, and (d) a nearby sounding from Montgomery, Alabama (KMGM), taken from the 0300 UTC RAP model initialization on 3 Mar 2012. Wind speeds are indicated on the sounding in meters per second. In (a)-(c), an oval indicates the region of debris and an arrow indicates the approximate direction of the environmental wind at the altitude shown in (d). Beam height at the center of the oval is approximately $3.4 \mathrm{~km}$, with a range of $\sim 59 \mathrm{~km}$.

although $\rho_{\mathrm{hv}}$ may not be as consistently low as when debris is associated with a small-radius vortex. In these events, a debris-consistent signature was not observed at low altitude outside the TDS-it is presumed that debris slowly sedimented out at too low of a density to substantially influence the polarimetric variables.

In a subset of cases ( $\sim 10 \%$ of TDS events), a signature has been observed that is apparently unique to TDS-producing supercell storms and has only been observed after a TDS has been observed for at least three or four volume scans (10-20 min). Evidence is presented here indicating that the signature represents debris fallout along the northwest flank of these storms. Thus, it has been named the debris fallout signature (DFS). A typical example is shown in Fig. 9, from an EF1 tornado in Alabama for which the genesis-to-demise period was reported to last $22 \mathrm{~min}$. A TDS first appeared at 0322 UTC, $7 \mathrm{~min}$ after reported tornadogenesis. By 0336 UTC, reduced $\rho_{\mathrm{hv}}$ values had not yet appeared outside the TDS at low levels (Figs. 9a,b), although aloft a streamer of lower $\rho_{\text {hv }}$ values was evident extending away from the TDS toward the northeast (Fig. 9c). This streamer was associated with $Z_{\mathrm{HH}}$ of approximately $50 \mathrm{dBZ}$ (Fig. 9d). By 0340 UTC, 3 min after tornado demise, the signature was well defined at base scan. Low $\rho_{\mathrm{hv}}$ values were located mostly north of the 


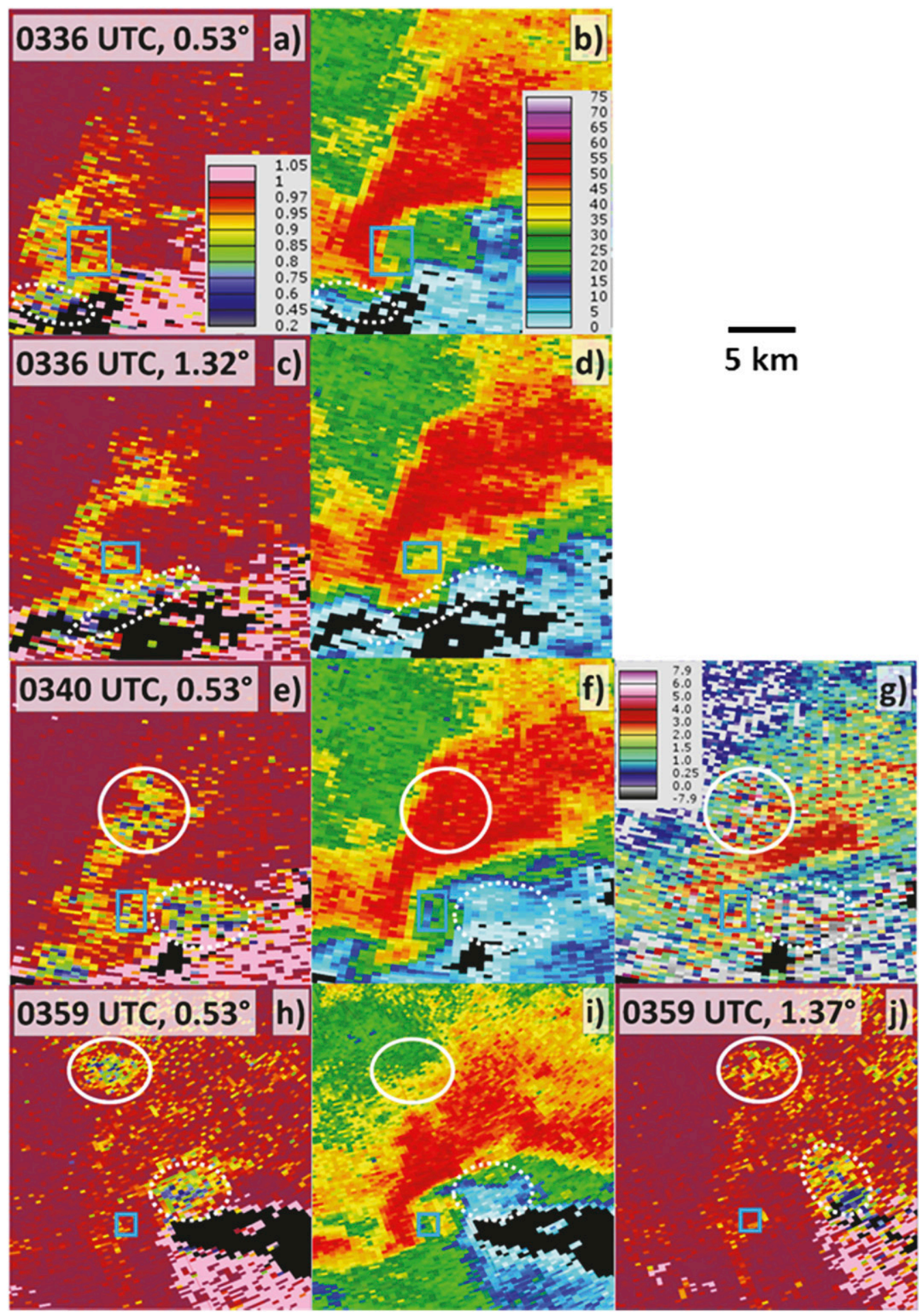

FIG. 9. Example of a DFS from KBMX on 3 Mar 2012. Each row contains images from the indicated time and elevation angle: (a),(c),(e),(h),(j) $\rho_{\mathrm{hv}},(\mathrm{b}),(\mathrm{d}),(\mathrm{f}),(\mathrm{i}) Z_{\mathrm{HH}}$, and (g) $Z_{\mathrm{DR}}$. Solid white circles indicate the DFS at 0340 and 0359 UTC; DFS aloft in (j) is slightly offset from DFS at base-scan level in (h) and (i). Blue squares show the region of maximum cyclonic rotation indicated by radial velocity. Dashed white circles indicate regions where $\rho_{\mathrm{hv}}$ is low as a result of a low signal-to-noise ratio. The vortex in (a) and (b) is centered approximately $59 \mathrm{~km}$ from the radar (altitude of $\sim 0.72 \mathrm{~km}$ ). The vortex in (c) and (d) is centered approximately $58 \mathrm{~km}$ from the radar (altitude of $\sim 1.56 \mathrm{~km})$. In (e)-(g), the DFS is centered approximately $49 \mathrm{~km}$ from the radar (altitude of $\sim 0.61 \mathrm{~km}$ ). In (h) and (i) the DFS is centered approximately $38 \mathrm{~km}$ from the radar (altitude of $\sim 0.46 \mathrm{~km}$ ). In (j), the residual area of debris aloft is centered approximately $40 \mathrm{~km}$ from the radar (altitude of $\sim 1.06 \mathrm{~km}$ ) 
remnant tornado (Fig. 9e), in an area of $Z_{\mathrm{HH}}$ between 45 and $55 \mathrm{dBZ}$ (Fig. 9f) and associated with noisy but predominantly near-zero $Z_{\mathrm{DR}}$ values (Fig. 9g). Spatiotemporal continuity of the signature persists through at least 0359 UTC (Fig. 9h), by which time it was located near the storm's northwest flank (Fig. 9i). Low $\rho_{\mathrm{hv}}$ values were becoming markedly less evident aloft (Fig. 9j), consistent with sedimentation. In this event, the signature was characterized by low $\rho_{\text {hv }}$ (Fig. 9e) and near-zero $Z_{\mathrm{DR}}$ (Fig. 9g), usually along the storm's northwest flank, although not associated with a low-level vortex.

This signature appears to represent debris that reaches base-scan level. It is not in the correct location to be categorized as a wake signature (Ryzhkov et al. 2005; Van Den Broeke et al. 2008). The reduction of base-scan $\rho_{\text {hv }}$ values commonly to $0.40-0.60$ is not consistent with most meteorological scatterers. Mixed-phase precipitation is precluded, given an environmental temperature at the altitude of the base-scan signature ( $~ 0.45 \mathrm{~km}$ by $0359 \mathrm{UTC}$ ) of approximately $18^{\circ} \mathrm{C}$ (not shown). Hail-growth regions may be characterized by low $\rho_{\text {hv }}$ values (e.g., Picca and Ryzhkov 2012), although the high temperature and location on the northwest flank of the storm (Figs. 9h,i,j) preclude this possibility. Although early in the signature's existence it was in a region favorable for hail (Figs. 9c,d), the coherent migration of the signature through time to low altitude along the storm's northwest flank also precludes this possibility. Biological scatter may have low $\rho_{\mathrm{hv}}$ values, but $Z_{\mathrm{DR}}$ tending near zero within the signature rules out most known bioscatter types (e.g., Van Den Broeke 2013). The signature was not in a typical location for smoke or chaff. Thus, debris emerges as the most likely type of scatterer present. Given the appearance of the DFS spatially separated from the TDS, it is speculated that DFS debris elements are small. This is consistent with uncommon reports of debris falling out in this general region well left of the tornado track (e.g., Snow et al. 1995). When vertical DFS characteristics were investigated across the sample of cases, in all but one case a debris-consistent polarimetric signature was observed to originate within the TDS, ascend to high altitude, appear later at successively lower elevation angles or the base scan, and then disappear first aloft and then in the base scan. Such sedimentation-consistent behavior is expected in falling debris. Ground-truth studies would be helpful to confirm particle identity within the signature.

Values of the radar variables were relatively consistent across the sample of DFSs observed. The $Z_{\mathrm{HH}}$ was typically greater than $30 \mathrm{~dB} Z$ in part of the DFS but could be as low as $20-25 \mathrm{~dB} Z$. The event shown in Fig. 9 was unusual, with small areas of $Z_{\mathrm{HH}}$ greater than
$50 \mathrm{~dB} Z$. The value of $Z_{\mathrm{HH}}$ within the DFS may depend on how much precipitation is mixed with debris. In these events, debris-associated $Z_{\mathrm{HH}}$ blended into the storm's northwest flank and did not appear nonmeteorological without use of the polarimetric variables, particularly $\rho_{\text {hv }}$ (e.g., Fig. 9). The $\rho_{\text {hv }}$ in the DFS most commonly ranged from 0.60 to 0.80 but in rare cases could be as low as $0.20-0.40$ or as high as 0.90 . Each DFS examined had at least one pixel with $\rho_{\mathrm{hv}}$ of less than 0.65 . The $Z_{\mathrm{DR}}$ within the DFS tended near $0 \mathrm{~dB}$ in all cases examined (e.g., Fig. 9g) -in fact, $Z_{\mathrm{DR}}$ was more consistently near $0 \mathrm{~dB}$ within the DFS than in the TDS for this sample of events.

Circumstances were briefly investigated that favor debris to spread out aloft or for a DFS to manifest along the northwest storm flank. Instability and shear variables did not appear strongly related to either mode of debris dispersion. MLCAPE derived from the Rapid Refresh assimilation/modeling system (RAP) was lower for cases in which debris spread out aloft $(p=0.037)$, although no other significant ambient variable associations were discovered. Each mode of debris dispersion was associated with an average TDS altitude that was higher than the average of the entire dataset $(p=0.0015$ for debris spreading out aloft; $p=0.0155$ for DFS events). Therefore, initially lofting debris to a higher altitude appears conducive to having the debris fall out in a concentrated plume or to be spread out downstream as it encounters stronger winds aloft.

\section{Summary and discussion}

Values of the polarimetric variables may vary widely between TDS events. Similar to the findings of Bodine et al. (2013), values of $Z_{\mathrm{HH}}$ increased and $\rho_{\mathrm{hv}}$ decreased with tornado intensity rating, likely because stronger tornadoes may pick up larger quantities of debris to higher altitude. Tornadoes responsible for very large reported property damage typically had low $\rho_{\mathrm{hv}}$ values. Higher $Z_{\mathrm{HH}}$ and lower $\rho_{\mathrm{hv}}$ values may indicate more violent tornadoes, particularly in distinguishing between EF0-2 and EF3 + events, but use of this finding in real time requires consideration of additional factors, including range to the TDS, land cover, and how the tornado may be interacting with nearby precipitation.

Among all TDS events, the probability of a radar scan containing a TDS increased through the tornado life cycle until tornado demise. Through tornado life cycles, the most statistically significant changes were for $Z_{\mathrm{HH}}$ to be highest during the mid-life cycle and for $\rho_{\text {hv }}$ to increase markedly after reported tornado demise. Thus, a possible interpretation of decreasing $Z_{\mathrm{HH}}$ and increasing $\rho_{\mathrm{hv}}$ is that a tornado is no longer actively lofting 
debris, although in some individual cases this is not true. For most TDS events examined, however, an increase in $\rho_{\text {hv }}$ to higher values within the TDS than were seen previously indicated that a tornado was no longer present. Fluctuations in $\rho_{\mathrm{hv}}$ values within TDSs were more common and more extreme than were $Z_{\mathrm{HH}}$ fluctuations.

If a TDS was observed in association with a given tornado, it typically appeared within $5 \mathrm{~min}$ of reported genesis and ended within $5 \mathrm{~min}$ after reported demise. In nearly $10 \%$ of cases, however, a TDS was manifest prior to reported genesis. It is unknown if these events represent an increase in the low-level wind field that is lofting debris, the presence of an unreported tornado, or incorrect times reported for tornadogenesis. If nowcasters see a TDS-consistent signature in the absence of a reported tornado, these results support issuance of a tornado warning if the parent storm may be tornado favorable and a low-level vortex is present. TDS occurrence was less predictable relative to reported tornado demise, because many TDSs disappeared well prior to reported demise and a subset of others persisted as long as $20 \mathrm{~min}$ afterward. The $\rho_{\mathrm{hv}}$ increased in a statistically significant way across the volume scan associated with reported tornado demise, and from this sample of events it appears to be the best variable to diagnose tornado demise.

The effects of land cover on TDS characteristics has been a topic of recent interest (e.g., Stelten and Wolf 2014; Van Den Broeke and Jauernic 2014). The median $Z_{\mathrm{HH}}$ value was relatively low over urban, grassland, and coniferous forest, and the median $\rho_{\mathrm{hv}}$ was relatively low over grassland and cropland. These results indicate that land cover has a relatively small effect on values of the polarimetric variables within TDSs. This is not universally the case, however-especially when a tornado is near a radar, in which case land-cover changes may produce noticeable changes in the radar variables. This may be especially true over urban land cover where large, heavy debris elements remain at relatively low altitude. Tornado intensity and percentage of TDSs associated with supercell storms over varying land-cover classes did not appear to strongly influence these results, although $\rho_{\text {hv }}$ values were more strongly related to tornado intensity than to land cover. Analysis of additional TDS events may provide more support for these preliminary findings, which are not statistically significant in this dataset.

Among all TDS datapoints at altitudes of less than $4.5 \mathrm{~km}$, TDS areal extent increased with altitude. The exception was for EF4/5 tornadoes, in which TDS areal extent decreased with altitude. From a nowcasting perspective, areal extent of the TDS may have some utility in diagnosing tornado intensity. Since exceptions were observed, however, these clues alone should not be used to diagnose tornado intensity.

The maximum altitude of TDS visibility was more strongly a function of tornado intensity than of land cover or ambient shear or instability. When a TDS is visible to high altitude, it still appears reasonable to suspect that a strong tornado may be on going (e.g., Bodine et al. 2013; Van Den Broeke and Jauernic 2014).

It is generally not evident where debris within a TDS goes. Debris may occasionally be observed beyond the TDS, and it is valuable for nowcasters to be aware of related signatures. Two primary modes of debris dispersion were noted, each in $\sim 10 \%$ of TDS cases examined and each associated with a TDS visible to relatively high altitude. In the first, debris spreads out downstream from the associated vortex, giving the impression that the TDS is larger than the associated vortex. In such cases, dispersion of debris was noted in the direction of the storm-relative wind at the altitude of dispersion. In the second mode, an apparently separate debris-consistent signature, denoted the DFS, becomes apparent near or along the northwest flank of the storm generally north of the TDS-associated vortex. A DFS typically appears late in the tornado life cycle and after a TDS has been visible for several volume scans. DFSs are characterized by $Z_{\mathrm{HH}}$ most commonly of $30-35 \mathrm{~dB} Z$, $\rho_{\mathrm{hv}}$ values from 0.60 to 0.80 , and $Z_{\mathrm{DR}}$ values consistently near $0 \mathrm{~dB}$.

Acknowledgments. The author is supported by an academic appointment at the University of NebraskaLincoln. Sabrina Jauernic is acknowledged for help with the radar analysis and assignment of land-cover classifications for each TDS event. Three anonymous reviewers provided comments that substantially strengthened the manuscript.

\section{REFERENCES}

Atkins, N. T., K. M. Butler, K. R. Flynn, and R. M. Wakimoto, 2014: An integrated damage, visual, and radar analysis of the 2013 Moore, Oklahoma, EF5 tornado. Bull. Amer. Meteor. Soc., 95, 1549-1561, doi:10.1175/BAMS-D-14-00033.1.

Bodine, D., M. R. Kumjian, A. J. Smith, R. D. Palmer, A. V. Ryzhkov, and P. L. Heinselman, 2011: High-resolution polarimetric observations of an EF-4 tornado on 10 May 2010 from OU-PRIME. Proc. 35th Conf. on Radar Meteorology, Pittsburgh, PA, Amer. Meteor. Soc., 4B.3. [Available online at https://ams.confex.com/ ams/35Radar/webprogram/Paper191661.html.]

,,- , R. D. Palmer, P. L. Heinselman, and A. V. Ryzhkov, 2013: Tornado damage estimation using polarimetric radar. Wea. Forecasting, 28, 139-158, doi:10.1175/WAF-D-11-00158.1. , R. D. Palmer, and G. Zhang, 2014: Dual-wavelength polarimetric radar analyses of tornadic debris signatures. J. Appl. Meteor. Climatol., 53, 242-261, doi:10.1175/ JAMC-D-13-0189.1. 
Bunkers, M. J., and M. A. Baxter, 2011: Radar tornadic debris signatures on 27 April 2011. Electron. J. Oper. Meteor., 12 (7). [Available online at http://www.nwas.org/ej/pdf/2011-ION1.pdf.]

Davies-Jones, R. P., D. W. Burgess, L. R. Lemon, and D. Purcell, 1978: Interpretation of surface marks and debris patterns from the 24 May 1973 Union City, Oklahoma tornado. Mon. Wea. Rev., 106, 12-21, doi:10.1175/1520-0493(1978)106<0012: IOSMAD $>2.0 . \mathrm{CO} ; 2$.

Dowell, D. C., C. R. Alexander, J. M. Wurman, and L. J. Wicker, 2005: Centrifuging of hydrometeors and debris in tornadoes: Radar-reflectivity patterns and wind-measurement errors. Mon. Wea. Rev., 133, 1501-1524, doi:10.1175/MWR2934.1.

Knox, J. A., and Coauthors, 2013: Tornado debris characteristics and trajectories during the 27 April 2011 Super Outbreak as determined using social media data. Bull. Amer. Meteor. Soc., 94, 1371-1380, doi:10.1175/BAMS-D-12-00036.1.

Kumjian, M. R., and A. V. Ryzhkov, 2008: Polarimetric signatures in supercell thunderstorms. J. Appl. Meteor. Climatol., 47, 1940-1961, doi:10.1175/2007JAMC1874.1.

Kurdzo, J. M., D. J. Bodine, B. L. Cheong, and R. D. Palmer, 2015: High-temporal resolution polarimetric X-band Doppler radar observations of the 20 May 2013 Moore, Oklahoma, tornado. Mon. Wea. Rev., 143, 2711-2735, doi:10.1175/ MWR-D-14-00357.1.

Lewellen, D. C., B. Gong, and W. S. Lewellen, 2008: Effects of finescale debris on near-surface tornado dynamics. J. Atmos. Sci., 65, 3247-3262, doi:10.1175/2008JAS2686.1.

Magsig, M. A., and J. T. Snow, 1998: Long-distance debris transport by tornadic thunderstorms. Part I: The 7 May 1995 supercell thunderstorm. Mon. Wea. Rev., 126, 1430-1449, doi:10.1175/1520-0493(1998)126<1430:LDDTBT>2.0.CO;2.

Picca, J., and A. Ryzhkov, 2012: A dual-wavelength polarimetric analysis of the 16 May 2010 Oklahoma City extreme hailstorm. Mon. Wea. Rev., 140, 1385-1403, doi:10.1175/ MWR-D-11-00112.1.

Ryzhkov, A. V., T. J. Schuur, D. W. Burgess, and D. S. Zrnić, 2005: Polarimetric tornado detection. J. Appl. Meteor., 44, 557-570, doi:10.1175/JAM2235.1.

Saari, M. D. W., R. M. Lawton, C. J. Schultz, and L. D. Carey, 2014: Early characteristics of the polarimetric tornadic debris signature associated with the 20 May 2013 Newcastle-Moore, Oklahoma, tornado. J. Oper. Meteor., 2, 110-114, doi:10.15191/nwajom.2014.0210.

Schultz, C. J., and Coauthors, 2012: Dual-polarization tornadic debris signatures part I: Examples and utility in an operational setting. Electron. J. Oper. Meteor., 13, 120-137. [Available online at http://www.nwas.org/ej/pdf/2012-EJ9.pdf.]
Schwarz, C. M., and D. W. Burgess, 2011: Supercell polarimetric signatures at X-band: Data from VORTEX2. Proc. 35th Conf. on Radar Meteorology, Pittsburgh, PA, Amer. Meteor. Soc., 7B.4. [Available online at https://ams.confex.com/ams/ 35Radar/webprogram/Paper191298.html.]

Smith, B. T., R. L. Thompson, J. S. Grams, and C. Broyles, 2012: Convective modes for significant severe thunderstorms in the contiguous United States. Part I: Storm classification and climatology. Wea. Forecasting, 27, 1114-1135, doi:10.1175/ WAF-D-11-00115.1.

Snow, J. T., A. L. Wyatt, A. K. McCarthy, and E. K. Bishop, 1995: Fallout of debris from tornadic thunderstorms: A historical perspective and two examples from VORTEX. Bull. Amer. Meteor. Soc., 76, 1777-1790, doi:10.1175/ 1520-0477(1995)076<1777:FODFTT >2.0.CO;2.

Stelten, S., and R. Wolf, 2014: Investigating the effect of land cover on the correlation between TDS heights and tornado strength and other TDS characteristics. Abstracts, 18th Annual Severe Storms and Doppler Radar Conf., Ankeny, IA, National Weather Association Central Iowa Chapter. [Available online at http://www.iowa-nwa.com/conference/files/2014_Agenda_ Lite.pdf.]

Tanamachi, R. L., H. B. Bluestein, J. B. Houser, S. J. Frasier, and K. M. Hardwick, 2012: Mobile, X-band, polarimetric Doppler radar observations of the 4 May 2007 Greensburg, Kansas, tornadic supercell. Mon. Wea. Rev., 140, 2103-2125, doi:10.1175/MWR-D-11-00142.1.

Van Den Broeke, M. S., 2013: Polarimetric radar observations of biological scatters in Hurricanes Irene (2011) and Sandy (2012). J. Atmos. Oceanic Technol., 30, 2754-2767, doi:10.1175/ JTECH-D-13-00056.1.

— , and S. T. Jauernic, 2014: Spatial and temporal characteristics of polarimetric tornadic debris signatures. J. Appl. Meteor. Climatol., 53, 2217-2231, doi:10.1175/JAMC-D-14-0094.1.

_ J. M. Straka, and E. N. Rasmussen, 2008: Polarimetric radar observations at low levels during tornado life cycles in a small sample of classic southern plains supercells. J. Appl. Meteor. Climatol., 47, 1232-1247, doi:10.1175/ 2007JAMC1714.1.

Wang, Y., and T. Yu, 2015: Novel tornado detection using an adaptive neuro-fuzzy system with S-band polarimetric weather radar. J. Atmos. Oceanic Technol., 32, 195-208, doi:10.1175/JTECH-D-14-00096.1.

WDTD, cited 2011: Dual-polarization radar training. National Weather Service Warning Decision Training Division. [Available online at http://www.wdtb.noaa.gov/courses/ dualpol/index.html.] 